Astron. Nachr. 000 (0000) 0, 000-000

\title{
Compact Groups of Galaxies in the Las Campanas Redshift Survey
}

\author{
S. S. Allam, Helwan, Cairo, Egypt \\ National Research Institute of Astronomy and Geophysics \\ D. L. TuCker, Batavia, Illinois, USA \\ Fermi National Accelerator Laboratory
}

Received ; accepted

\begin{abstract}
We have recently extracted a catalog of compact groups of galaxies (CGs) from the Las Campanas Redshift Survey. This catalog of Las Campanas Compact Groups (LCCGs) contains 76 CGs with a median redshift of $z_{\text {med }} \approx 0.08$. The physical properties of these CGs are similar to those from the Hickson (1982) and the Barton et al. (1996) catalogs. Here, we present an atlas of our catalog and briefly describe its general properties.
\end{abstract}

Key words: catalogs - surveys - galaxies: galaxies

\section{Introduction}

Perhaps over half of all galaxies lie within groups containing 3 - 20 members (Tully 1987); yet, due to the difficulty of discerning them from the field, groups of galaxies are, as a whole, not as well studied as larger galaxy systems. Compact groups (CGs), however, defined by their small number of members $(<10)$, their compactness (typical intra-group separations of a galaxy diameter or less), and their relative isolation (intra-group separations $\ll$ groupfield separations) are more readily identifiable; Stephan's Quintet is a beautiful and noteworthy example.

The best known catalog of such systems is that of the Hickson Compact Groups (HCGs; Hickson 1982), a sample comprising 100 groups selected from the red prints of the Palomar Observatory Sky Survey (POSS). Due to their high densities (equivalent to those at the centers of rich clusters) and low velocity dispersions $\left(\sim 200 \mathrm{~km} \mathrm{~s}^{-1}\right)$, HCGs represent an environment where interactions, tidally triggered activity, and galaxy mergers are expected to be more frequent than in most other surroundings. Therefore, the HCG catalog has served as an excellent laboratory for the study of the effects of immediate environment on a galaxy's morphology and stellar composition.

Nonetheless, it is necessary to compare and extend the results gleaned from the HCG catalog using more modern catalogs. The Las Campanas Compact Group (LCCG) catalog, which we extracted from the Las Campanas Redshift Survey (LCRS; Shectman et al. 1996), contains 76 CGs and is among the small handful of new catalogs which is extending our knowledge of CG environments. Since it is based upon a modern galaxy redshift survey which in turn is based upon CCD photometry, the LCCG catalog enjoys certain advantages over the HCG, one of the most important being the homogeneity of the sample: e.g., one can compare the properties of compact group galaxies from the LCRS with those of loose group galaxies from the LCRS with those of field galaxies from the $L C R S$. Systematic errors obtained from comparing heterogeneous samples are thus eliminated [see, for example, the results of Allam et al. (1999)].

Here, we present both the LCCG catalog itself and a "postage stamp" atlas of its member groups. We have organized the paper as follows: in Sec. (2.) we overview the LCRS, in Sec. (3.) we describe the "friends-of-friends" percolation algorithm used in extracting the CG catalog from the LCRS, in Sec. (4.) we present the Las Campanas Compact Group (LCCG) catalog and atlas, and in Sec. (5.) we analyze the properties of the LCCGs and compare them to the properties of two other catalogs. Finally, we summarize our results in Sec. (6.).

\section{The Data}

The LCRS is an $R$-band selected survey; it extends to a redshift of 0.2 and contains 26,418 galaxy redshifts of which 23,697 lie within the official geometric and photometric limits of the survey. Accurate photometry and sky positions for program objects were extracted from CCD drift scans obtained on the Las Campanas Swope 1-m

${ }^{*}$ Visiting Scientist, Fermi National Accelerator Laboratory 
telescope; spectroscopy was performed at the Las Campanas Du Pont 2.5-m telescope, originally via a 50-fiber Multi-Object Spectrograph (MOS), and later via a 112-fiber MOS.

For observing efficiency, all the fibers were used, but each MOS field was observed only once. Hence, the LCRS is a collection of 50-fiber fields (with nominal apparent magnitude limits of $16.0 \leq R<17.3$ ) and 112-fiber fields (with nominal apparent magnitude limits of $15.0 \leq R<17.7$ ). Observing each field only once, however, creates a problem for finding compact groups: the protective tubing of the individual fibers prevented the spectroscopic observation of both members of galaxy pairs within 55 arcsec of each other. Therefore, many galaxies in CG environments are missing from the LCRS redshift catalog. We have partially circumvented this problem by assigning each of the $\sim$ 1,000 "missing" LCRS galaxies the redshift of its nearest neighbor (convolved with a gaussian of $\sigma=200 \mathrm{~km} \mathrm{~s}^{-1}$ ). Hence, on the small angular scales necessary for compact group selection, the LCRS falls somewhere between a 2D sky survey and a fully 3D redshift survey.

\section{Compact Group Selection}

We have selected CGs based on physical extent rather than angular size. We applied a friends-of-friends algorithm (Ramella et al. 1989) to extract a sample of CGs systems in the LCRS (hereafter, LCCGs). In constructing the LCCG catalog, we have considered only those LCRS galaxies within the official geometric and photometric borders of the survey; furthermore, we have limited this sample to galaxies having redshifts in the range $7,500 \mathrm{~km} \mathrm{~s}^{-1} \leq$ $c z_{\mathrm{cmb}}<50,000 \mathrm{~km} \mathrm{~s}^{-1}$ and luminosities in the range $-22.5 \leq M_{R}-5 \log h<-17.5$. To avoid CG member incompleteness at the extremal distances of the sample, only groups within the range $10,000 \mathrm{~km} \mathrm{~s}^{-1} \leq c z_{\mathrm{cmb}}<$ $45,000 \mathrm{~km} \mathrm{~s}^{-1}$ were admitted into the final LCCG catalog.

We identify CGs as linked sets of neighboring galaxies. A seed galaxy $i$ is selected which has not yet been classified as either a CG member or an isolated galaxy. Every other non-classified galaxy $j$ in the survey sample is then tested to see if it lies within a projected separation $D_{\mathrm{L}}$ and a velocity difference $V_{\mathrm{L}}$ of the seed galaxy:

$$
\begin{aligned}
& D_{i j}=2 D_{\text {ave }} \sin \left(\Theta_{i j} / 2\right) \leq D_{\mathrm{L}} \text { and } \\
& V_{i j}=c \times\left|z_{i}-z_{j}\right| \leq V_{\mathrm{L}},
\end{aligned}
$$

where $D_{\text {ave }} \equiv\left(D\left(z_{i}\right)+D\left(z_{j}\right)\right) / 2$ and $\Theta_{i j}$ is the angular separation between the two galaxies. The comoving distances $D(z)$ are calculated assuming $q_{0}=0.5$ and $H_{0}=h \times 100 \mathrm{~km} \mathrm{~s}^{-1} \mathrm{Mpc}^{-1}\left(h \equiv H_{0} / 100 \mathrm{~km} \mathrm{~s}^{-1} \mathrm{Mpc}^{-1}\right)$, and the redshifts $z$ are corrected for motion relative to the dipole moment of the cosmic microwave background (CMB; Lineweaver et al. 1996).

If no companions are found within $D_{\mathrm{L}}$ and $V_{\mathrm{L}}$ of the seed galaxy, it is assigned "isolated" status and another seed galaxy is sought. If companions are found, they are added along with the seed galaxy to a list of CG members forming a new group. In turn, the surroundings of each of these companions are combed for the next level of "friends." This loop is repeated until no further companions are located, and the process is begun again by pursuing another seed galaxy. The CG catalog is complete only once every galaxy in the redshift sample has been classified as either "isolated" or "grouped." Only those groups containing three or more members are included in the final catalog.

Following Barton et al. (1996), we chose values of $D_{\mathrm{L}}$ and $V_{\mathrm{L}}$ which would produce a catalog of LCRS compact groups which had physical properties similar to those of the HCGs. Our definition was as follows:

- low membership: number of group members $N_{\text {tot }} \geq 3$ galaxies,

- compact: projected inter-galaxy separation of $D_{i j} \leq D_{\mathrm{L}}=50 h^{-1} \mathrm{kpc}(\sim 1$ galaxy diameter $)$, and

- isolated: inter-galaxy velocity difference $V_{i j} \leq V_{\mathrm{L}}=1000 \mathrm{~km} \mathrm{~s}^{-1}$

Using these criteria a total of 76 CGs were identified by a systematic search of the LCRS, each CG having three or more member galaxies. The total number of galaxies contained witin the set of LCCGs is 253 .

\section{Compact Group Catalog}

We list the properties of all the LCCGs in Table (2). Tabulated are the following:

Column (1): a running identification number.

Columns (2 \& 3): the group's right ascension and declination (1950 coordinates).

Column (4): $z_{\mathrm{cmb}}$, the group's redshift.

Column (5): $N_{\text {tot }}$, the number of galaxies in the group.

Column (6): $R_{\mathrm{p}}$, the group's mean pairwise separation.

Column (7): $R_{\mathrm{h}}$, the group's harmonic radius. 
Column (8): $L_{\text {tot }}$, the group's LCRS $R$-band total luminosity, corrected for galaxies outside the LCRS's photometric limits. (Since several other group catalogs have photometry in the de Vaucouleurs $B_{0}$ band, it is useful to note this rough conversion relation: $L_{\mathrm{LCRS}} \approx 1.1 L_{B_{0}}$.)

Column (9): $L_{\text {rat }}$, the ratio by which the observed group members' summed luminosities must be multiplied to obtain $L_{\text {tot }}$.

Column (10): $\Theta_{\mathrm{G}}$, the angular radius of the smallest circle which includes all the group's members.

Column (11): $R_{\mathrm{G}}$, the physical radius of the smallest circle which includes all the group's members.

Column (11): the type of field in which the group sits ("1" = a 50-fiber field, "2" = a 112-fiber field).

Column (12): A column to reference any applicable notes for the group:

a: the group's barycenter is closer than $2 R_{\mathrm{p}}$ to a slice edge.

b: the group contains at least one 55 arcsec "orphan" with a mock redshift.

Further details in how these properties were measured can be found in Tucker et al. (2000), which describes a catalog of LCRS loose groups.

\subsection{Group Members}

Table 3 lists the members of each group. Column (1) provides a group member identification, where the letter "a" represents the brightest group member, the letter "b" the second brightest group member, and so on. Columns ( 2 \& 3) list the group member's right ascension and declination in 1950.0 coordinates. The LCRS $R$-band isophotal magnitude for the group member is given in Column (4), and its velocity is listed in Col. (5). As noted earlier, in generating the LCLG catalog, each 55-arcsec "orphan" was assigned a mock velocity equal to the gaussianconvolved velocity of its nearest neighbor (a gaussian of $\sigma=200 \mathrm{~km} \mathrm{~s}^{-1}$ was used); this mock velocity is listed in this column for 55-arcsec "orphan" group members.

Using the $R$-band magnitude of the LCRS $\left(m_{R}\right)$ and the measured spectroscopic redshift, a photometric redshift relation was also derived. A first order least square fit was made to the data $\left(0.03 \pm 0.001 \times m_{R}-0.43 \pm 0.028\right.$; see Fig. 1). This magnitude-redshift relation was then re-applied to each CG member with a mock velocity; the resulting photometric redshift, $z_{\text {photo }}$, is listed in Col. (6).

Finally, in Col. (7), we provide a qualitative description of the group member's spectrum:

c: a "continuum" spectrum (i.e., a spectrum with no strong emission line features),

e: a spectrum containing strong emission lines,

b: a spectrum which shows both absorption lines (as in a continuum spectrum) and weak emission lines, and

m: a 55-arcsec "orphan" with a mock redshift.

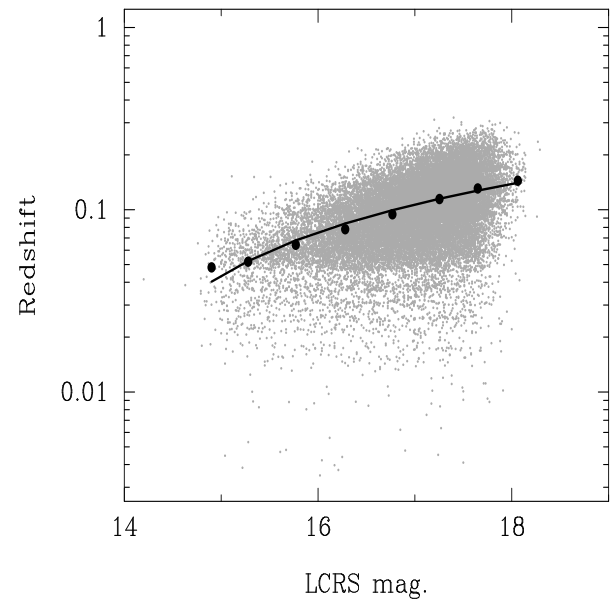

Fig. 1: $R$-band magnitude vs. measured (i.e., spectroscopic) redshift for galaxies in the LCRS spectroscopic sample. The solid line represents the linear fit. The filled circles denote the median redshift for a given magnitude bin. 


\subsection{The LCCG Atlas}

For each of the 76 LCCGs, we have created an atlas image with the following characteristics (see Fig. ??):

- Each atlas image is a 100 pixel $\times 100$ pixel "postage stamp" with a resolution of $1.7 \operatorname{arcsec}$ pixel ${ }^{-1}$ (obtained from the Digitized Sky Survey, courtesy of SkyView).

- Within each atlas image, the identified group members are labelled by their lowercase letter designation (a, b, c, ...; see Table 3).

- Within each atlas image, the group barycenter is marked by a " $\times$ ".

Note that in many of these fields there are galaxies which are not identified as group members. These galaxies are either outside the photometric limits of the LCRS redshift catalog, and/or members of a loose group or rich cluster in which the LCCG is embedded, and/or 55 arcsec "orphans" which have been accidentally excluded from the LCCG.

\section{Properties of LCCGs}

\subsection{Compact Groups and Large-Scale Structure}

The cone diagrams in Figures (2A-D) show the positions of the LCCGs and their individual group members for the LCRS Northern and Southern Galactic Cap regions. Note that the distribution of LCCGs is non-random: from comparison with cone diagrams of all LCRS galaxies (Shectman et al. 1996), it is clear that the LCCGs (and the LCCG members) trace many of the same large-scale structures, albeit much more sparsely. This is not too surprising, since one would not expect to find CGs where where there are no galaxies. On the other hand, this adds fuel to the arguments that CGs are not truly isolated, and that many of them may just be chance alignments along the line-of-sight of large-scale filamentary structures and/or that they may form continuously within loose groups or rich clusters of galaxies (Mamon 1986, Hernquist et al. 1995, Diaferio et al. 1994). [Due to the significant differences between the star formation properties of loose group galaxies and compact group galaxies (e.g., Allam et al. 1999), the latter scenario - in which CGs are real substructures within loose groups and rich clusters and not merely chance alignments - is the more likely.]
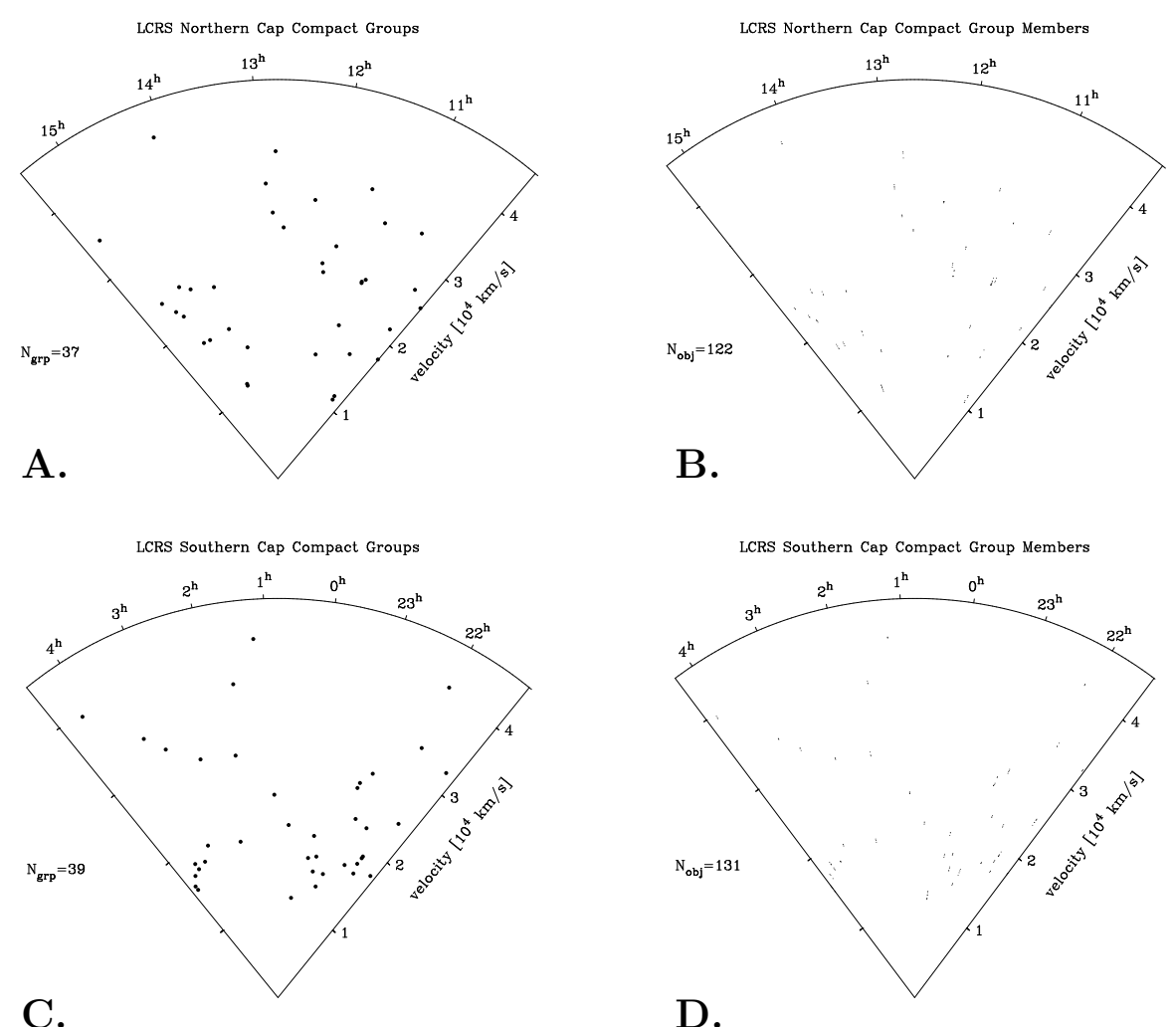

Fig. 2: The cone diagrams of LCCGs in the Northern (top), and Southern (bottom) Galactic Cap. 


\subsection{Environments of Compact Groups}

We have examined the regions around the LCCGs visually using images from the Digitized Sky Survey and by using the NED database. We have also correlated the positions of these CGs with the catalog of loose groups of galaxies by Tucker et al. (2000). From these investigations, we have found the following:

- 1/3 of LCCGs are "isolated," lying outside group or cluster environments,

- $\sim 1 / 3$ of LCCGs are embedded within poor loose groups of galaxies, and

- $\sim 1 / 3$ of LCCGs are embedded within rich, Abell-class clusters.

\subsection{Comparison with Other Compact Group Catalogs}

It is instructive to compare the LCCG catalog with CG catalogs derived from other galaxy redshift surveys, like that by Barton et al. (1996) (the RSCG catalog), and those based upon sky surveys (e.g., the HCG catalog). Table 1 and Figure 3 summarize the properties of the 76 LCCGs, the 92 HCGs, and the 89 RSCGs. Note that, for the most part, all three catalogs contain systems with very similar physical properties. There are, however, two areas in which the catalogs differ. First, the HCG catalog contains a smaller fraction of triplets than do either the LCCG or the RSCG catalogs, a result of a different population threshold originally for the HCG catalog. (Groups were only admitted to the HCG catalog from the POSS plates if they contained at least four galaxies; groups were only admitted to the LCLG and the RSCG samples if they contained at least three galaxies.) Second, the LCCG catalog is much deeper on average than either of the other two CG catalogs, which is due to the depth of the LCRS itself.

Table 1: Compact Groups Physical Parameters

\begin{tabular}{lcccccc}
\hline Parameter & \multicolumn{2}{c}{ LCCGs } & \multicolumn{2}{c}{ HCGs } & \multicolumn{2}{c}{ RSCGs } \\
& mean & median & mean & median & mean & median \\
\hline$N_{\text {tot }}$ & $3.28 \pm 0.069$ & 3 & $4.15 \pm 0.10$ & 4 & $3.65 \pm 0.14$ & 3 \\
$z_{\mathrm{cmb}}$ & $0.08 \pm 0.003$ & 0.079 & $0.03 \pm 0.002$ & 0.03 & $0.013 \pm 0.001$ & 0.014 \\
$\theta_{\mathrm{G}}[\mathrm{deg}]$ & $0.016 \pm 0.0009$ & 0.0131 & $0.0601 \pm 0.0005$ & 0.0483 & $\ldots$ & $\ldots$ \\
$R_{\mathrm{G}}\left[h^{-1} \mathrm{kpc}\right]$ & $26.31 \pm 1.00$ & 26.0 & $24.69 \pm 1.53$ & 21.76 & $29.99 \pm 1.63$ & 26.90 \\
$R_{\mathrm{p}}\left[h^{-1} \mathrm{Mpc}\right]$ & $0.05 \pm 0.002$ & 0.05 & $0.05 \pm 0.02$ & 0.05 & $\ldots$ & $\ldots$ \\
$R_{\mathrm{h}}\left[h^{-1} \mathrm{Mpc}\right]$ & $0.05 \pm 0.002$ & 0.05 & $0.06 \pm 0.004$ & 0.05 & $\ldots$ & $\ldots$ \\
$L\left[10^{10} L_{\odot} h^{-2}\right]$ & $7.24 \pm 1.08$ & $7.4^{*}$ & $7.4 \pm 0.54^{*}$ & 6.5 & $\ldots$ & $\ldots$ \\
\hline * The HCG total luminosities were converted to the LCRS $R$-band assuming $L_{\mathrm{LCS}} \approx 1.1 L_{B_{0}}$
\end{tabular}

\section{Conclusions}

We have applied a "friends-of-friends" group identification algorithm (Huchra \& Geller 1982) to the Las Campanas Redshift Survey (LCRS) in order to produce a catalog of compact groups (CGs) physically similar to those of the Hickson (1982) catalog. We defined these Las Campanas Compact Groups (LCCGs) by the following criteria: $N_{\text {tot }} \geq 3$ galaxies, with projected separations of $D \leq D_{\mathrm{L}}=50 h^{-1} \mathrm{kpc}(\sim 1$ galaxy diameter $)$ and velocity differences of $V \leq V_{\mathrm{L}}=1000 \mathrm{~km} \mathrm{~s}^{-1}$. As a result, we extracted a catalog of 76 LCCGs, each containing 3 or more galaxies. The total number of galaxies in the LCCG sample is 253. All the LCCGs contain at least 1 spectroscopically determined redshift; 23 contain 2 or more. The mean redshift of LCCGs is $z_{\mathrm{cmb}} \sim 0.08$.

We have evaluated the physical characteristics of the LCCGs and have found the following:

- The main physical properties of the LCCGs (membership, linear size, total group luminosity) are similar to those of HCGs.

- The median redshift is $\sim 0.08$, more than twice that of either the HCG or the Barton et al. (1996) catalogs.

- Only about one-third of LCCGs are isolated; the remaining two-thirds are embedded within loose groups or clusters.

We therefore conclude that the catalog of LCCGs is a useful addition to the study of compact groups. We further conclude that, although a significant minority of compact groups appear to be truly isolated, the majority of these systems are in reality dense substructures within larger galaxy associations (e.g., loose groups and rich clusters). 

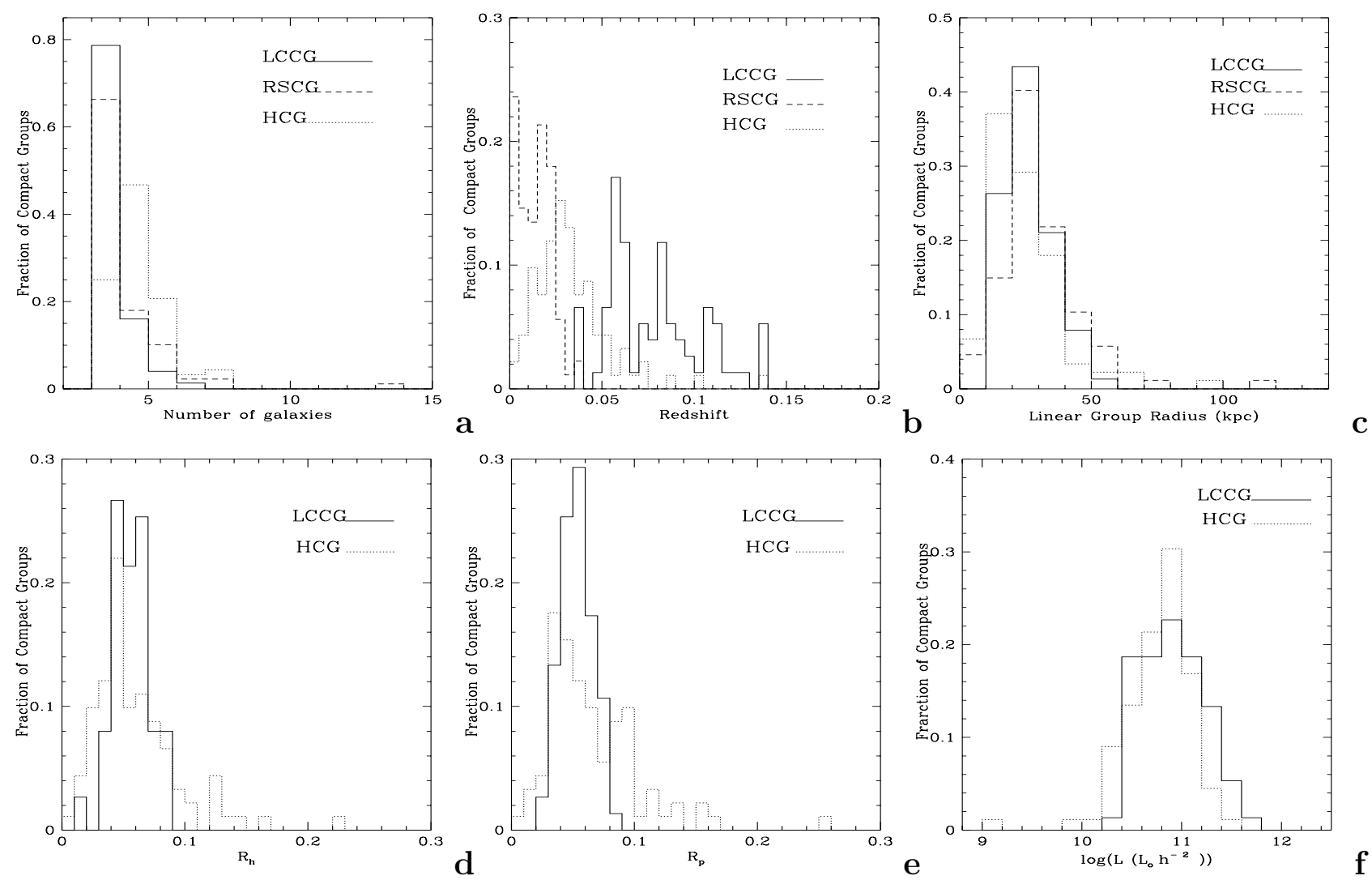

Fig. 3: (a) The distribution of group populations, $N_{\mathrm{tot}}$, (b) the distribution of group redshifts, $z_{\mathrm{cmb}}$, (c) the distribution of linear group radii, $R_{\mathrm{G}}$, (d) the distribution of harmonic radii, $R_{\mathrm{h}}$, (e) the distribution of mean pairwise separations, $R_{\mathrm{p}}$, and (f) the distribution of total group luminosities, $L_{\text {tot }}$, for LCCGs (solid line), RSCGs (dashed line), and HCGs (dotted line). Each distribution is normalized to the total number of CGs in each sample. For (f), the luminosities of the HCGs were converted to LCRS $R$-band luminosities assuming $L_{\mathrm{LCRS}} \approx 1.1 L_{B_{0}}$.

Acknowledgements. This research has made use of the NASA/IPAC Extragalactic Database (NED), which is operated by the Jet Propulsion Laboratory, at the California Institute of Technology, under contract with the National Aeronautics and Space Administration. The Digitized Sky Survey was produced at the Space Telescope Science Institute under US government grant NAGW-2166.

This work was supported in part by the US Department of Energy under contract No. DE-AC02-76CH03000.

\section{References}

Allam, S., Tucker, D., Lin,H., Hashimoto, Y.: 1999, Astrophys. J. 522, L89

Barton, E., Geller, M., Ramella, M., Marzke, R., de Costa, L.: 1996, Astron. J. 112, 871

Diaferio, A., Geller, M.J., Ramella, M.: 1994, Astron. J. 107, 868

Hernquist, L., Katz, N., Weinberg, D.H.: 1995, Astrophys. J., 442, 57

Hickson, P.: 1982, Astrophys. J., 225, 382

Mamon, G.A.: 1986, Astrophys. J., 307, 426

Ramella, M., Geller, M.J., Huchra, J.P.: 1989, Astrophys. J., 344, 57

Shectman, S., Landy, S., Oemler A., Tucker, D., Lin, H., Kirshner, R., Schechter, P.: 1996, Astrophys. J., 470,172

Tucker, D., Oemler, A., Hashimoto, Y., Shectman, S., Kirshner, R., Lin, H., Landy,S., Schechter, P., Allam, S.: 2000, Astrophys. J. Suppl., submitted

Addresses of the authors:

Allam, S., Helwan, Cairo, Egypt, sallam@fnal.gov

Tucker, D., Batavia, Illinois, USA, dtucker@fnal.gov 
Table 2: The main properties of the Las Campanas Compact Groups (LCCGs).

\begin{tabular}{|c|c|c|c|c|c|c|c|c|c|c|c|}
\hline \multirow{3}{*}{$\begin{array}{l}\mathrm{N} \\
(1)\end{array}$} & DEC & \multirow[t]{2}{*}{$z_{c m b}$} & \multirow[t]{2}{*}{$N_{t o t}$} & $R_{P}$ & $R_{h}$ & L & $L_{\text {rat }}$ & \multirow{3}{*}{$\begin{array}{c}\theta_{G} \\
(10)\end{array}$} & \multirow{3}{*}{$\begin{array}{c}R_{G} \\
\operatorname{Mpc} h^{-1} \\
(11)\end{array}$} & \multirow{3}{*}{$\begin{array}{l}\text { Type } \\
\text { (12) }\end{array}$} & \multirow{3}{*}{$\begin{array}{c}\text { Notes } \\
\text { (13) }\end{array}$} \\
\hline & $(1950)$ & & & \multicolumn{2}{|c|}{$\operatorname{Mpc} h^{-1}$} & \multirow{2}{*}{$\begin{array}{c}L_{\odot} h^{-2} \\
(8)\end{array}$} & & & & & \\
\hline & (3) & $(4)$ & $(5)$ & (6) & $(7)$ & & (9) & & & & \\
\hline 01 & $002204.59-453416.0$ & 0.0666 & 3 & 0.04 & 0.05 & $0.63 \mathrm{E} 11$ & 1.91 & 0.0132 & 0.0411 & 2 & $\mathrm{~b}$ \\
\hline 02 & $004549.54-421707.0$ & 0.0780 & 4 & 0.07 & 0.08 & $0.18 \mathrm{E} 12$ & 6.12 & 0.0245 & 0.0875 & 1 & $\mathrm{~b}$ \\
\hline 03 & $010407.90-443511.9$ & 0.1384 & 3 & 0.04 & 0.05 & $0.15 \mathrm{E} 12$ & 3.01 & 0.0064 & 0.0370 & 2 & $\mathrm{~b}$ \\
\hline 04 & $012120.48-392951.7$ & 0.1217 & 3 & 0.06 & 0.06 & $0.18 \mathrm{E} 12$ & 4.64 & 0.0112 & 0.0585 & 2 & $\mathrm{~b}$ \\
\hline 05 & $013907.37-445102.5$ & 0.0948 & 3 & 0.05 & 0.05 & $0.55 \mathrm{E} 11$ & 2.17 & 0.0121 & 0.0511 & 2 & $\mathrm{~b}$ \\
\hline 06 & $020024.78-450118.8$ & 0.0620 & 3 & 0.04 & 0.04 & $0.29 \mathrm{E} 11$ & 1.98 & 0.0114 & 0.0332 & 2 & $\mathrm{~b}$ \\
\hline 07 & $022701.50-444042.5$ & 0.0972 & 3 & 0.06 & 0.08 & $0.16 \mathrm{E} 12$ & 4.34 & 0.0142 & 0.0615 & 1 & $\mathrm{~b}$ \\
\hline 08 & $025011.91-390640.7$ & 0.1050 & 3 & 0.04 & 0.05 & $0.12 \mathrm{E} 12$ & 2.46 & 0.0095 & 0.0436 & 2 & $\mathrm{~b}$ \\
\hline 09 & $025317.43-392633.2$ & 0.0646 & 3 & 0.05 & 0.06 & $0.71 \mathrm{E} 11$ & 1.79 & 0.0185 & 0.0561 & 2 & $\mathrm{~b}$ \\
\hline 10 & $031832.39-422633.9$ & 0.1138 & 3 & 0.06 & 0.07 & $0.16 \mathrm{E} 12$ & 5.02 & 0.0131 & 0.0646 & 2 & $\mathrm{~b}$ \\
\hline 11 & $032027.75-413201.7$ & 0.0601 & 4 & 0.05 & 0.04 & $0.38 \mathrm{E} 11$ & 4.31 & 0.0261 & 0.0741 & 2 & $\mathrm{~b}$ \\
\hline 12 & $033026.56-381806.3$ & 0.0585 & 3 & 0.02 & 0.03 & $0.63 \mathrm{E} 11$ & 2.72 & 0.0092 & 0.0256 & 2 & $\mathrm{~b}$ \\
\hline 13 & $033322.62-385010.9$ & 0.0611 & 4 & 0.07 & 0.08 & $0.89 \mathrm{E} 11$ & 3.43 & 0.0294 & 0.0846 & 2 & $\mathrm{~b}$ \\
\hline 14 & $035748.08-412800.8$ & 0.0581 & 3 & 0.07 & 0.08 & $0.67 \mathrm{E} 11$ & 1.72 & 0.0293 & 0.0808 & 2 & $a b$ \\
\hline 15 & $041701.69-421712.5$ & 0.0554 & 3 & 0.05 & 0.05 & $0.28 \mathrm{E} 11$ & 1.86 & 0.0197 & 0.0520 & 2 & $\mathrm{~b}$ \\
\hline 16 & $041708.65-421916.7$ & 0.0537 & 3 & 0.07 & 0.07 & $0.60 \mathrm{E} 11$ & 1.92 & 0.0281 & 0.0720 & 2 & $\mathrm{~b}$ \\
\hline 17 & $041934.59-452210.0$ & 0.1391 & 3 & 0.03 & 0.04 & $0.24 \mathrm{E} 12$ & 4.67 & 0.0049 & 0.0283 & 2 & $\mathrm{~b}$ \\
\hline 18 & $100430.11-052243.2$ & 0.0598 & 3 & 0.03 & 0.03 & $0.67 \mathrm{E} 11$ & 2.37 & 0.0085 & 0.0241 & 2 & $\mathrm{~b}$ \\
\hline 19 & $100452.96-052635.4$ & 0.0854 & 3 & 0.04 & 0.05 & $0.12 \mathrm{E} 12$ & 2.53 & 0.0120 & 0.0465 & 2 & $\mathrm{~b}$ \\
\hline 20 & $101728.72-033733.5$ & 0.0718 & 3 & 0.04 & 0.04 & $0.57 \mathrm{E} 11$ & 2.33 & 0.0109 & 0.0364 & 2 & $\mathrm{~b}$ \\
\hline 21 & $102042.80-053723.6$ & 0.0897 & 3 & 0.04 & 0.04 & $0.25 \mathrm{E} 12$ & 7.17 & 0.0094 & 0.0380 & 1 & $\mathrm{~b}$ \\
\hline 22 & $102621.32-025902.0$ & 0.0370 & 3 & 0.05 & 0.04 & $0.23 \mathrm{E} 11$ & 3.41 & 0.0306 & 0.0555 & 2 & $\mathrm{~b}$ \\
\hline 23 & $102738.03-025440.0$ & 0.0385 & 3 & 0.06 & 0.06 & $0.34 \mathrm{E} 11$ & 3.42 & 0.0296 & 0.0557 & 2 & \\
\hline 24 & $104330.75-021843.3$ & 0.1095 & 3 & 0.05 & 0.06 & $0.86 \mathrm{E} 11$ & 2.78 & 0.0105 & 0.0502 & 2 & $\mathrm{~b}$ \\
\hline 25 & $104405.36-112200.7$ & 0.0555 & 3 & 0.04 & 0.04 & $0.30 \mathrm{E} 11$ & 2.01 & 0.0158 & 0.0418 & 2 & $\mathrm{~b}$ \\
\hline 26 & $110948.15-025310.1$ & 0.0837 & 3 & 0.04 & 0.04 & $0.11 \mathrm{E} 12$ & 2.56 & 0.0105 & 0.0399 & 2 & $\mathrm{~b}$ \\
\hline 27 & $111214.34-060540.1$ & 0.0818 & 4 & 0.05 & 0.06 & $0.32 \mathrm{E} 12$ & 6.10 & 0.0158 & 0.0588 & 1 & $\mathrm{~b}$ \\
\hline 28 & $111249.75-033804.0$ & 0.0824 & 5 & 0.08 & 0.07 & $0.91 \mathrm{E} 11$ & 2.62 & 0.0299 & 0.1123 & 2 & $\mathrm{~b}$ \\
\hline 29 & $111319.86-111730.0$ & 0.1069 & 3 & 0.05 & 0.05 & $0.13 \mathrm{E} 12$ & 2.85 & 0.0124 & 0.0581 & 2 & $a b$ \\
\hline 30 & $111831.24-022830.8$ & 0.0636 & 3 & 0.03 & 0.03 & $0.29 \mathrm{E} 11$ & 1.52 & 0.0088 & 0.0263 & 2 & $\mathrm{~b}$ \\
\hline 31 & $113218.84-122149.7$ & 0.1177 & 3 & 0.07 & 0.08 & $0.20 \mathrm{E} 12$ & 3.81 & 0.0142 & 0.0718 & 2 & $\mathrm{~b}$ \\
\hline 32 & $113736.45-030456.0$ & 0.0500 & 3 & 0.03 & 0.01 & $0.32 \mathrm{E} 11$ & 2.73 & 0.0120 & 0.0289 & 2 & $\mathrm{~b}$ \\
\hline 33 & $114838.04-024501.6$ & 0.0923 & 3 & 0.04 & 0.04 & $0.14 \mathrm{E} 12$ & 3.49 & 0.0089 & 0.0367 & 2 & $\mathrm{~b}$ \\
\hline 34 & $115536.56-021640.4$ & 0.0815 & 5 & 0.07 & 0.06 & $0.91 \mathrm{E} 11$ & 1.58 & 0.0226 & 0.0838 & 2 & $a b$ \\
\hline 35 & $115819.59-112412.0$ & 0.0849 & 3 & 0.05 & 0.06 & $0.41 \mathrm{E} 11$ & 1.79 & 0.0136 & 0.0523 & 2 & $\mathrm{~b}$ \\
\hline 36 & $121429.53-025520.5$ & 0.1083 & 5 & 0.06 & 0.06 & $0.19 \mathrm{E} 12$ & 3.11 & 0.0165 & 0.0781 & 2 & $\mathrm{~b}$ \\
\hline 37 & $124035.33-115726.3$ & 0.0971 & 3 & 0.03 & 0.04 & $0.11 \mathrm{E} 12$ & 2.86 & 0.0067 & 0.0288 & 2 & $\mathrm{~b}$ \\
\hline 38 & $124700.38-060439.3$ & 0.1259 & 3 & 0.06 & 0.06 & $0.51 \mathrm{E} 12$ & 8.04 & 0.0124 & 0.0661 & 1 & $\mathrm{~b}$ \\
\hline 39 & $125023.74-114844.0$ & 0.1028 & 3 & 0.04 & 0.05 & $0.66 \mathrm{E} 11$ & 2.72 & 0.0098 & 0.0443 & 2 & $\mathrm{~b}$ \\
\hline 40 & $125527.92-115254.3$ & 0.1141 & 4 & 0.05 & 0.06 & $0.15 \mathrm{E} 12$ & 4.01 & 0.0136 & 0.0674 & 2 & $\mathrm{~b}$ \\
\hline 41 & $133856.05-115054.0$ & 0.0521 & 3 & 0.03 & 0.04 & $0.61 \mathrm{E} 11$ & 2.55 & 0.0134 & 0.0335 & 2 & $\mathrm{~b}$ \\
\hline 42 & $135903.99-112402.2$ & 0.0378 & 3 & 0.05 & 0.06 & $0.24 \mathrm{E} 11$ & 2.62 & 0.0303 & 0.0561 & 2 & $\mathrm{~b}$ \\
\hline 43 & $135907.57-111948.3$ & 0.0385 & 4 & 0.06 & 0.04 & $0.38 \mathrm{E} 11$ & 2.62 & 0.0424 & 0.0801 & 2 & $a b$ \\
\hline 44 & $135909.10-033016.2$ & 0.0777 & 3 & 0.04 & 0.04 & $0.57 \mathrm{E} 11$ & 1.66 & 0.0118 & 0.0420 & 2 & $\mathrm{~b}$ \\
\hline 45 & $135939.23-120037.5$ & 0.0609 & 3 & 0.04 & 0.05 & $0.25 \mathrm{E} 11$ & 1.74 & 0.0146 & 0.0421 & 2 & $\mathrm{~b}$ \\
\hline 46 & $140502.25-022121.1$ & 0.1398 & 3 & 0.05 & 0.05 & $0.22 \mathrm{E} 12$ & 3.84 & 0.0087 & 0.0506 & 2 & $\mathrm{~b}$ \\
\hline 47 & $142418.60-031620.8$ & 0.0802 & 3 & 0.04 & 0.04 & $0.37 \mathrm{E} 11$ & 1.48 & 0.0100 & 0.0367 & 2 & $\mathrm{~b}$ \\
\hline 48 & $143203.24-113640.3$ & 0.0597 & 3 & 0.05 & 0.06 & $0.40 \mathrm{E} 11$ & 1.58 & 0.0189 & 0.0533 & 2 & $\mathrm{~b}$ \\
\hline 49 & $143434.73-033309.2$ & 0.0829 & 3 & 0.05 & 0.05 & $0.90 \mathrm{E} 11$ & 3.49 & 0.0118 & 0.0447 & 1 & $\mathrm{~b}$ \\
\hline 50 & $143954.52-034310.0$ & 0.0594 & 4 & 0.04 & 0.04 & $0.56 \mathrm{E} 11$ & 1.88 & 0.0154 & 0.0434 & 2 & $a b$ \\
\hline
\end{tabular}


Table 2: The main properties of LCCGs (continued)

\begin{tabular}{|c|c|c|c|c|c|c|c|c|c|c|c|}
\hline \multirow{3}{*}{$\begin{array}{l}\mathrm{N} \\
(1)\end{array}$} & \multirow{2}{*}{ RA } & \multirow{3}{*}{$\begin{array}{c}z_{c m b} \\
(4)\end{array}$} & \multirow{3}{*}{$\begin{array}{c}N_{t o t} \\
(5)\end{array}$} & \multirow{2}{*}{\multicolumn{2}{|c|}{$\begin{array}{lr}R_{P} & R_{h} \\
\mathrm{Mpc} & h^{-1}\end{array}$}} & \multirow{3}{*}{$\begin{array}{c}\mathrm{L} \\
L_{\odot} h^{-2} \\
\quad(8)\end{array}$} & \multirow{3}{*}{$\begin{array}{l}L_{\text {rat }} \\
\\
(9)\end{array}$} & \multirow{3}{*}{$\begin{array}{l}\theta_{G} \\
(10)\end{array}$} & \multirow{3}{*}{$\begin{array}{c}R_{G} \\
\mathrm{Mpc} h^{-1} \\
(11)\end{array}$} & \multirow{3}{*}{$\begin{array}{l}\text { Type } \\
\text { (12) }\end{array}$} & \multirow{3}{*}{$\begin{array}{l}\text { Notes } \\
\text { (13) }\end{array}$} \\
\hline & & & & & & & & & & & \\
\hline & $(2)$ & & & $(6)$ & $(7)$ & & & & & & \\
\hline 51 & $144553.00-030252.0$ & 0.0721 & 4 & 0.05 & 0.05 & $0.38 \mathrm{E} 11$ & 1.43 & 0.0153 & 0.0512 & 2 & $\mathrm{~b}$ \\
\hline 52 & $145056.82-024221.6$ & 0.0751 & 3 & 0.04 & 0.04 & $0.32 \mathrm{E} 11$ & 1.42 & 0.0114 & 0.0395 & 2 & $\mathrm{~b}$ \\
\hline 53 & $150213.21-111740.0$ & 0.0812 & 3 & 0.03 & 0.04 & $0.50 \mathrm{E} 11$ & 1.66 & 0.0092 & 0.0339 & 2 & $\mathrm{~b}$ \\
\hline 54 & $151228.56-023525.1$ & 0.1144 & 3 & 0.06 & 0.06 & $0.18 \mathrm{E} 12$ & 2.89 & 0.0110 & 0.0545 & 2 & $\mathrm{~b}$ \\
\hline 55 & $211547.19-420153.5$ & 0.1086 & 3 & 0.07 & 0.07 & $0.71 \mathrm{E} 11$ & 2.38 & 0.0141 & 0.0668 & 2 & $\mathrm{~b}$ \\
\hline 56 & $211831.69-454208.5$ & 0.0825 & 6 & 0.04 & 0.04 & $0.11 \mathrm{E} 12$ & 1.94 & 0.0143 & 0.0536 & 2 & $\mathrm{~b}$ \\
\hline 57 & $211932.20-394404.4$ & 0.0589 & 3 & 0.06 & 0.07 & $0.50 \mathrm{E} 11$ & 1.96 & 0.0242 & 0.0674 & 2 & $a b$ \\
\hline 58 & $214003.50-454158.0$ & 0.0564 & 3 & 0.04 & 0.05 & $0.19 \mathrm{E} 11$ & 2.54 & 0.0173 & 0.0465 & 2 & $\mathrm{~b}$ \\
\hline 59 & $214926.48-420948.6$ & 0.0635 & 3 & 0.07 & 0.08 & $0.32 \mathrm{E} 11$ & 1.73 & 0.0270 & 0.0806 & 2 & $\mathrm{~b}$ \\
\hline 60 & $214947.88-421019.3$ & 0.0627 & 3 & 0.04 & 0.04 & $0.26 \mathrm{E} 11$ & 1.76 & 0.0146 & 0.0431 & 2 & $\mathrm{~b}$ \\
\hline 61 & $215706.40-442956.7$ & 0.1369 & 3 & 0.05 & 0.05 & $0.24 \mathrm{E} 12$ & 5.06 & 0.0087 & 0.0499 & 2 & $\mathrm{~b}$ \\
\hline 62 & $215718.48-392451.9$ & 0.0598 & 3 & 0.05 & 0.05 & $0.31 \mathrm{E} 11$ & 2.10 & 0.0206 & 0.0583 & 2 & $\mathrm{~b}$ \\
\hline 63 & $220303.62-382038.4$ & 0.1107 & 3 & 0.05 & 0.06 & $0.73 \mathrm{E} 11$ & 2.77 & 0.0113 & 0.0546 & 2 & $\mathrm{~b}$ \\
\hline 64 & $220517.85-441943.5$ & 0.0738 & 3 & 0.03 & 0.04 & $0.61 \mathrm{E} 11$ & 2.42 & 0.0103 & 0.0352 & 2 & $\mathrm{~b}$ \\
\hline 65 & $220810.33-454143.2$ & 0.0574 & 3 & 0.06 & 0.06 & $0.38 \mathrm{E} 11$ & 2.49 & 0.0225 & 0.0611 & 2 & $a b$ \\
\hline 66 & $223251.14-414522.2$ & 0.0749 & 3 & 0.05 & 0.06 & $0.97 \mathrm{E} 11$ & 2.24 & 0.0134 & 0.0462 & 2 & $\mathrm{~b}$ \\
\hline 67 & $223558.12-414101.0$ & 0.0935 & 4 & 0.05 & 0.01 & $0.91 \mathrm{E} 11$ & 2.77 & 0.0124 & 0.0520 & 2 & $\mathrm{~b}$ \\
\hline 68 & $224237.18-451955.3$ & 0.0886 & 4 & 0.06 & 0.06 & $0.14 \mathrm{E} 12$ & 3.60 & 0.0163 & 0.0651 & 2 & $\mathrm{~b}$ \\
\hline 69 & $224720.97-421213.0$ & 0.0862 & 3 & 0.05 & 0.05 & $0.60 \mathrm{E} 11$ & 2.33 & 0.0138 & 0.0540 & 2 & $\mathrm{~b}$ \\
\hline 70 & $224745.66-453741.4$ & 0.0507 & 4 & 0.05 & 0.06 & $0.69 \mathrm{E} 11$ & 3.82 & 0.0263 & 0.0640 & 2 & $\mathrm{~b}$ \\
\hline 71 & $225913.84-413306.9$ & 0.0451 & 3 & 0.06 & 0.06 & $0.31 \mathrm{E} 11$ & 2.40 & 0.0282 & 0.0617 & 2 & $\mathrm{~b}$ \\
\hline 72 & $231637.14-421528.2$ & 0.0503 & 4 & 0.03 & 0.03 & $0.73 \mathrm{E} 11$ & 4.85 & 0.0124 & 0.0299 & 2 & $\mathrm{~b}$ \\
\hline 73 & $231750.01-420411.4$ & 0.0562 & 3 & 0.03 & 0.03 & $0.13 \mathrm{E} 12$ & 4.44 & 0.0116 & 0.0310 & 2 & $\mathrm{~b}$ \\
\hline 74 & $233322.26-385126.5$ & 0.0638 & 3 & 0.02 & 0.03 & $0.49 \mathrm{E} 11$ & 2.55 & 0.0080 & 0.0239 & 2 & $\mathrm{~b}$ \\
\hline 75 & $233536.46-384448.7$ & 0.0550 & 3 & 0.06 & 0.07 & $0.55 \mathrm{E} 11$ & 2.73 & 0.0259 & 0.0678 & 2 & $\mathrm{~b}$ \\
\hline 76 & $235944.84-441649.3$ & 0.0387 & 7 & 0.06 & 0.06 & $0.81 \mathrm{E} 11$ & 3.07 & 0.0375 & 0.0711 & 2 & $a b$ \\
\hline (1) & \multicolumn{3}{|c|}{ Running group ID number. } & $(8)$ & \multicolumn{7}{|c|}{ Luminosity in the LCRS $R$-band } \\
\hline$(2)$ & \multicolumn{3}{|c|}{ Right ascension } & $(9)$ & \multicolumn{7}{|c|}{ Observed-to-total luminosity corr } \\
\hline$(3)$ & \multicolumn{3}{|l|}{ Declination } & $(10)$ & \multicolumn{7}{|c|}{ Radius of the smallest circle including all members (in deg) } \\
\hline (4) & \multicolumn{3}{|l|}{ Redshift } & $(11)$ & \multicolumn{7}{|c|}{ Radius of the smallest circle including all members (in Mpc) } \\
\hline$(5)$ & \multicolumn{3}{|c|}{ Number of group member galaxies } & $(12)$ & \multicolumn{7}{|c|}{ The type of CG (see text) } \\
\hline (6) & \multicolumn{3}{|c|}{ Mean pairwise separation } & $(13)$ & \multicolumn{7}{|c|}{ Comments } \\
\hline$(7)$ & \multicolumn{11}{|l|}{ Harmonic radius } \\
\hline
\end{tabular}


Table 3: Compact Group Members

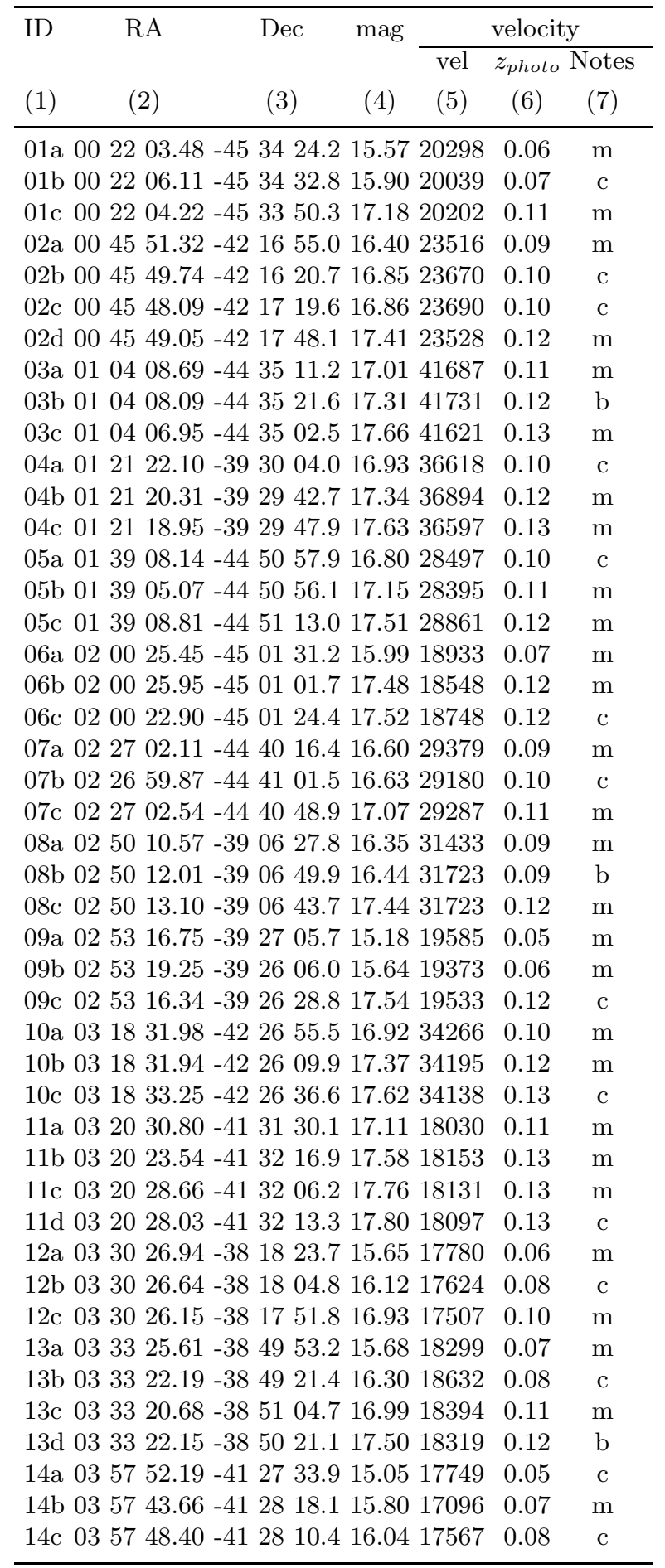

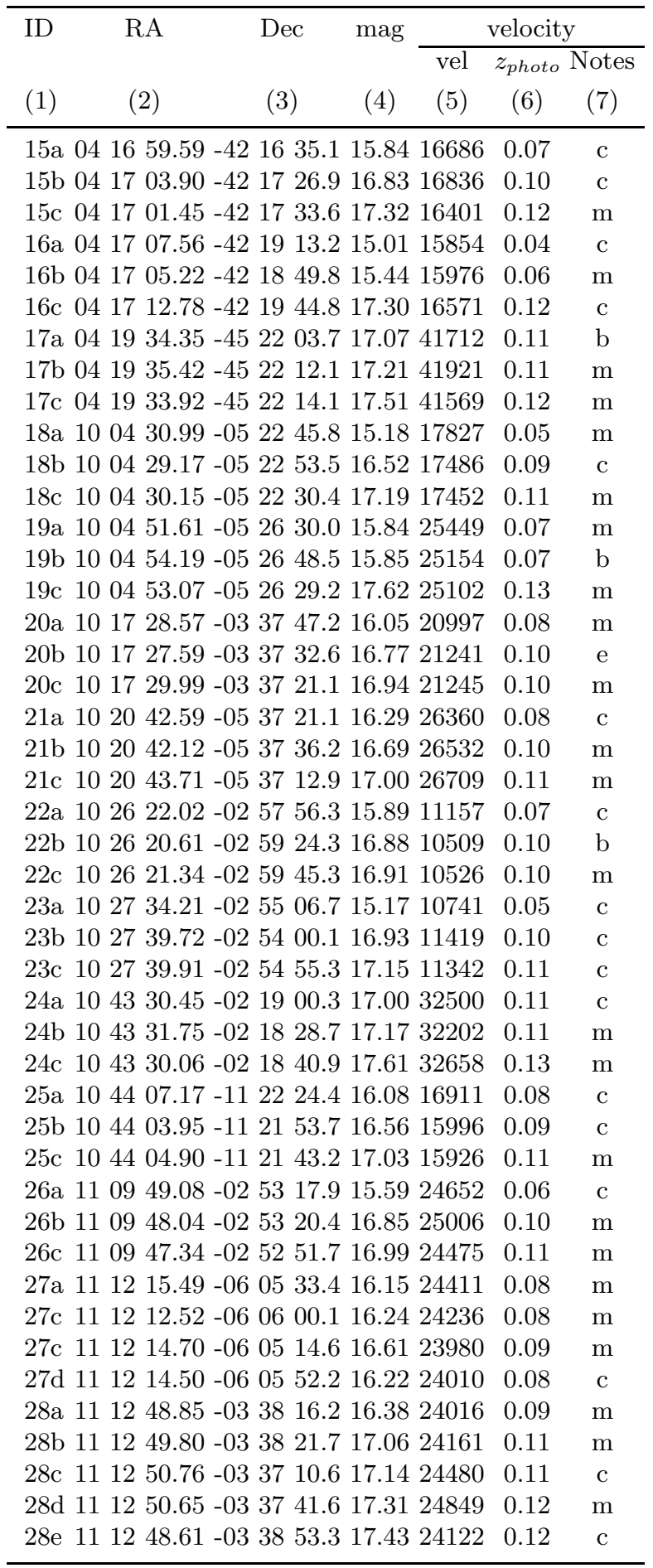


Table 3: Continued

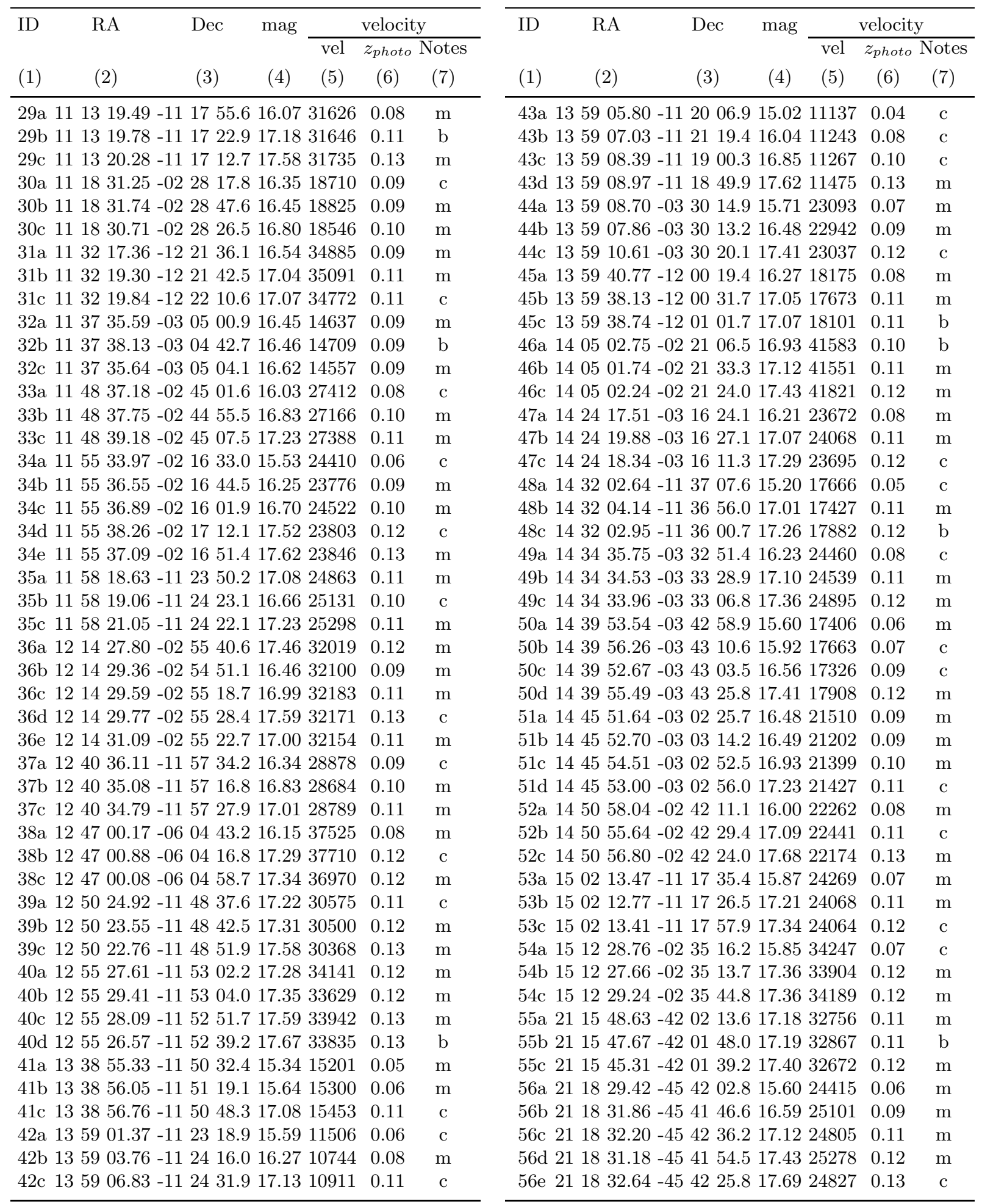


Table 3: Continued

\begin{tabular}{|c|c|c|c|c|c|}
\hline \multirow{3}{*}{$\begin{array}{l}\text { ID } \\
\text { (1) }\end{array}$} & \multirow{3}{*}{$\begin{array}{l}\text { RA } \\
(2)\end{array}$} & \multirow{3}{*}{$\begin{array}{c}\text { mag } \\
(4)\end{array}$} & \multicolumn{3}{|c|}{ velocity } \\
\hline & & & vel & $z_{\text {photo }}$ & Notes \\
\hline & & & $(5)$ & $(6)$ & $(7)$ \\
\hline $56 f$ & $\begin{array}{llllll}21 & 18 & 32.68 & -45 & 42 & 05.0\end{array}$ & 17.30 & 25189 & 0.12 & $\mathrm{~m}$ \\
\hline $57 \mathrm{a}$ & $211935.50-394351.7$ & 15.11 & 17949 & 0.05 & $\mathrm{c}$ \\
\hline $57 \mathrm{~b}$ & $211928.20-394412.1$ & 17.02 & 17945 & 0.11 & $\mathrm{c}$ \\
\hline $57 \mathrm{c}$ & $\begin{array}{llllll}21 & 19 & 32.80 & -39 & 44 & 09.7\end{array}$ & 17.56 & 17754 & 0.12 & $\mathrm{~m}$ \\
\hline $58 \mathrm{a}$ & $214001.77-454130.3$ & 16.99 & 17126 & 0.11 & $\mathrm{~b}$ \\
\hline $58 \mathrm{~b}$ & $\begin{array}{llllll}21 & 40 & 03.28 & -45 & 42 & 04.0\end{array}$ & 17.25 & 16997 & 0.11 & $\mathrm{~m}$ \\
\hline $58 \mathrm{c}$ & $\begin{array}{llllll}21 & 40 & 05.42 & -45 & 42 & 18.9\end{array}$ & 17.57 & 17185 & 0.12 & $\mathrm{e}$ \\
\hline $59 \mathrm{a}$ & $214924.19-420906.1$ & 15.79 & 19209 & 0.07 & $\mathrm{~m}$ \\
\hline $59 b$ & $\begin{array}{llllll}21 & 49 & 28.94 & -42 & 10 & 27.5\end{array}$ & 17.15 & 19573 & 0.11 & $\mathrm{~m}$ \\
\hline $59 c$ & $214926.06-42 \quad 0948.2$ & 17.41 & 19042 & 0.12 & $\mathrm{c}$ \\
\hline $60 \mathrm{a}$ & $\begin{array}{llllll}21 & 49 & 50.48 & -42 & 10 & 25.0\end{array}$ & 16.55 & 19122 & 0.09 & $\mathrm{~b}$ \\
\hline $60 \mathrm{~b}$ & $214947.44 \quad-42 \quad 1022.2$ & 16.72 & 18965 & 0.10 & $\mathrm{~m}$ \\
\hline $60 \mathrm{c}$ & $214945.95 \quad-42 \quad 1011.5$ & 17.11 & 18982 & 0.11 & $\mathrm{~m}$ \\
\hline $61 \mathrm{a}$ & $215707.77-442955.0$ & 17.07 & 41262 & 0.11 & $\mathrm{~m}$ \\
\hline $61 b$ & $215706.65-442957.4$ & 17.33 & 41173 & 0.12 & $\mathrm{c}$ \\
\hline $61 c$ & $215704.86-442957.6$ & 17.52 & 41332 & 0.12 & $\mathrm{~m}$ \\
\hline $62 \mathrm{a}$ & $\begin{array}{llllll}21 & 57 & 18.12 & -39 & 25 & 23.7\end{array}$ & 16.42 & 18309 & 0.09 & $\mathrm{c}$ \\
\hline $62 b$ & $215718.65-392410.6$ & 16.70 & 18172 & 0.10 & $\mathrm{~b}$ \\
\hline $62 c$ & $2157 \quad 18.69-392459.7$ & 16.96 & 18055 & 0.11 & $\mathrm{~m}$ \\
\hline $63 \mathrm{a}$ & $\begin{array}{llllll}22 & 03 & 03.83 & -38 & 20 & 38.3\end{array}$ & 17.30 & 33284 & 0.12 & $\mathrm{c}$ \\
\hline $63 \mathrm{~b}$ & $\begin{array}{llllll}22 & 03 & 04.58 & -38 & 20 & 54.2\end{array}$ & 17.49 & 33719 & 0.12 & $\mathrm{~m}$ \\
\hline $63 \mathrm{c}$ & $\begin{array}{llllll}22 & 03 & 02.44 & -38 & 20 & 22.2\end{array}$ & 17.50 & 33303 & 0.12 & $\mathrm{~m}$ \\
\hline $64 \mathrm{a}$ & $220518.01-441949.6$ & 15.85 & 22193 & 0.07 & $\mathrm{~m}$ \\
\hline $64 b$ & $\begin{array}{llllll}22 & 05 & 16.11 & -44 & 19 & 39.3\end{array}$ & 16.90 & 22510 & 0.10 & $\mathrm{~m}$ \\
\hline $64 \mathrm{c}$ & $22 \quad 0519.46-441941.7$ & 17.59 & 22327 & 0.13 & $\mathrm{c}$ \\
\hline $65 \mathrm{a}$ & $\begin{array}{llllll}22 & 08 & 07.18 & -45 & 41 & 15.1\end{array}$ & 16.13 & 17521 & 0.08 & $\mathrm{~b}$ \\
\hline $65 b$ & $2208 \quad 10.97 \quad-454142.0$ & 16.49 & 17371 & 0.09 & $\mathrm{~m}$ \\
\hline $65 c$ & $22 \quad 08 \quad 12.72 \quad-45 \quad 42 \quad 10.9$ & 17.16 & 17361 & 0.11 & $\mathrm{c}$ \\
\hline $66 \mathrm{a}$ & $223249.87-414541.3$ & 15.48 & 22652 & 0.06 & $\mathrm{~m}$ \\
\hline $66 \mathrm{~b}$ & $22 \quad 3253.52-414518.0$ & 16.28 & 22605 & 0.08 & $\mathrm{c}$ \\
\hline $66 \mathrm{c}$ & $22 \quad 3250.09-414508.6$ & 16.49 & 22807 & 0.09 & $\mathrm{~m}$ \\
\hline $67 a$ & $223557.07-414039.6$ & 16.99 & 28506 & 0.11 & $\mathrm{~m}$ \\
\hline $67 \mathrm{~b}$ & $223558.24-414122.0$ & 17.04 & 27996 & 0.11 & $\mathrm{~m}$ \\
\hline $67 \mathrm{c}$ & 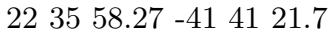 & 17.09 & 28332 & 0.11 & $\mathrm{c}$ \\
\hline $67 d$ & $223558.94-414044.0$ & 17.37 & 28199 & 0.12 & $\mathrm{~m}$ \\
\hline (1) & Compact group memb & & & & \\
\hline$(2)$ & Right ascension & & & & \\
\hline (3) & Declination & & & & \\
\hline (4) & $R$-band magnitude & & & & \\
\hline (5) & Velocity & & & & \\
\hline (6) & $z_{\text {photo }}$ & & & & \\
\hline (7) & Notes & & & & \\
\hline
\end{tabular}

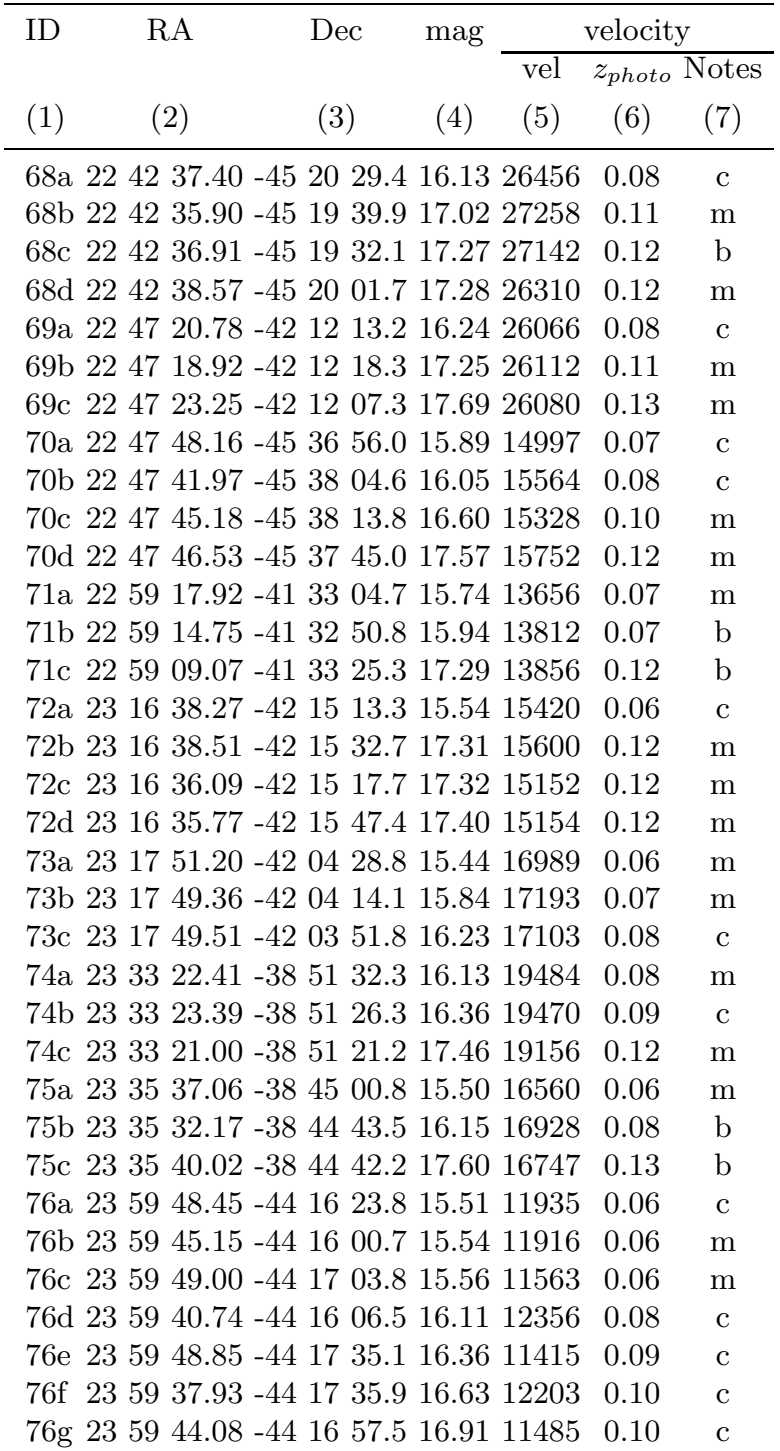


This figure "fig4a.gif" is available in "gif" format from: http://arxiv.org/ps/astro-ph/0006155v1 
This figure "fig4b.gif" is available in "gif" format from: http://arxiv.org/ps/astro-ph/0006155v1 
This figure "fig4c.gif" is available in "gif" format from: http://arxiv.org/ps/astro-ph/0006155v1 
This figure "fig4d.gif" is available in "gif" format from: http://arxiv.org/ps/astro-ph/0006155v1 